\title{
Regulation of chronic inflammatory and immune processes by extracellular vesicles
}

\author{
Paul D. Robbins, Akaitz Dorronsoro, and Cori N. Booker
}

Department of Metabolism and Aging, The Scripps Research Institute-Florida, Jupiter, Florida, USA.

\begin{abstract}
Almost all cell types release extracellular vesicles (EVs), which are derived either from multivesicular bodies or from the plasma membrane. EVs contain a subset of proteins, lipids, and nucleic acids from the cell from which they are derived. EV factors, particularly small RNAs such as miRNAs, likely play important roles in cell-to-cell communication both locally and systemically. Most of the functions associated with EVs are in the regulation of immune responses to pathogens and cancer, as well as in regulating autoimmunity. This Review will focus on the different modes of immune regulation, both direct and indirect, by EVs. The therapeutic utility of EVs for the regulation of immune responses will also be discussed.
\end{abstract}

\section{Introduction}

Extracellular vesicles (EVs) are comprised of both microvesicles, released from the plasma membrane by shedding, and nanovesicles or exosomes, generated by reverse budding of multivesicular bodies. These different types of vesicles are characterized predominantly by their size, with exosomes ranging from 30 to 100 $\mathrm{nm}$ and microvesicles usually being larger than $200 \mathrm{~nm}(1-4)$. Although their contents likely differ, both small and large vesicles are enriched for a subset of diverse proteins, mRNAs (5), and noncoding RNAs, such as microRNAs (miRNAs), which are derived from the parental cells (6). There also have been reports of extrachromosomal DNA present in certain types of EVs $(4,7)$.

EVs have a variety of reported functions, and many of their better documented activities are associated with some form of immune regulation (7). EVs from both immune and nonimmune cells, such as mesenchymal stem cells (MSCs) (8) and endothelial cells (ECs), contribute to antigen-specific and nonspecific immune regulation. Depending upon the context and vesicle type, EVs can stimulate or suppress the immune responses to infections with viruses and microbial pathogens as well as cancer. They also likely play a role in modulating inflammatory and autoimmune diseases, such as arthritis, diabetes, and lupus (9). Given their ability to modulate immune responses, EVs have tremendous potential as therapeutic agents for treating a variety of human diseases and disorders, including reducing inflammation, treating autoimmune diseases and cancer, and stimulating antipathogen immune responses. The focus of this Review will be on the role of both endogenous and exogenous EVs, primarily exosomes, in regulating immune responses and how these EVs can be used therapeutically for regulating the immune system.

\section{Immune regulation by antigen-presenting} cell EVs

Antigen-presenting cells (APCs), including DCs, macrophages, and $\mathrm{B}$ cells, regulate immune responses through direct interac-

Conflict of interest: P.D. Robbins receives funding support from Aldabra Biosciences and has filed patents on therapeutic uses of extracellular vesicles.

Reference information: J Clin Invest. 2016;126(4):1173-1180. doi:10.1172/JCI81131. tions with $\mathrm{CD}^{+}$and $\mathrm{CD} 8^{+} \mathrm{T}$ cells and other immune cell types, such as NK and NKT cells. Cell surface proteins, including MHC class I and II molecules; costimulatory molecules, such as B7 family members, including CD80, CD86, and programmed death-1 (PD-1); and adhesion molecules, such as ICAM, mediate the regulation of immune function by APCs (10-12). Because exosomes are formed by reverse budding of the multivesicular body, the extracellular domains of these and other proteins on the surface of the APCs are exposed functionally on the surface of the vesicles. The larger microvesicles released from the plasma membrane also carry a similar pattern of membrane proteins involved in $\mathrm{T}$ cell regulation. APC release of EVs with a similar pattern of surface immune regulatory proteins supports the hypothesis that APCs can modulate $T$ cell and other immune cell activity at a distance via EVs. In addition, the fact that DCs and B cells increase the release of exosomes following cognate $\mathrm{T}$ cell interactions also suggests that EVs are important for conferring part of the immunoregulatory effects of these cell types (13-15).

Multiple groups have reported that EVs from APCs can present peptide/MHC complexes to T cells. This can be mediated through several distinct mechanisms (Figure 1). Without internalization, EVs can bind to the surface of APCs, where they are well positioned to interact directly with $\mathrm{T}$ cells, a process termed cross-dressing (Figure 1A and ref. 16). In this context, costimulatory molecules present on the APC can interact with the T cell, while the EVs present the antigen epitope in the context of $\mathrm{MHC}$ class II (17). The fact that mature APCs release EVs with higher levels of adhesion molecules and MHC class II than immature APCs allows these vesicles bind efficiently with target APCs to activate T cells (17-19). However, MHC class II-deficient APCs were less efficient than WT APCs for activation of T cells, suggesting that presentation of peptide by MHC class II on the APC also contributes to cross-dressing (17).

Follicular DCs (FDCs) are an example of a cell type that clearly regulates T cells through cross-dressing. FDCs are nonphagocytic and do not synthesize MHC class II molecules but instead capture MHC class $\mathrm{II}^{+}$EVs derived from follicular B cells (20). Because FDCs are nonphagocytic, the captured EVs likely remain on the 

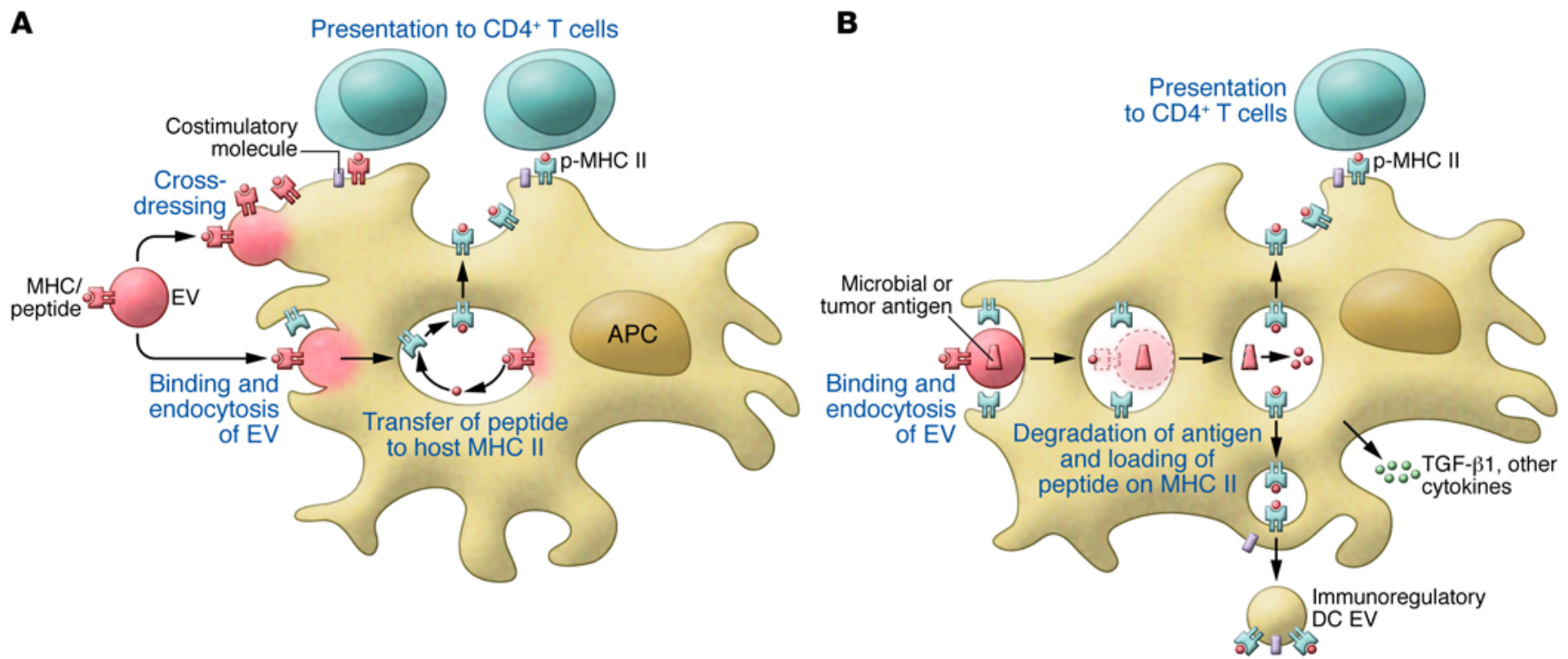

Figure 1. Regulation of immune responses by professional APCs. Professional APCs, such as DCs, present MHC class I and II complexes with peptides (p-MHC) that are derived from captured exosomes. (A) EVs retained on the APC surface present their p-MHC complexes directly to T cells, where costimulatory molecules and other regulatory molecules can be provided by the APC (cross-dressing). The EVs also can be internalized, allowing them to transfer their peptides to MHC molecules of the host APCs. Endogenous antigens can be processed in a similar manner, and the resulting epitopes are loaded onto MHC class II molecules. Host MHC class II complexed with EV-derived peptides (p-MHC II) are transported to the cell surface for presentation to T cells. (B) Alternatively, EV interaction with and uptake by APCs can lead to increased production of cytokines, such as TCF- $\beta 1$, and release of APC-derived EVs carrying $\mathrm{p}-\mathrm{MHC}$ II that are able to regulate antigen-specific immune responses. For simplicity, only MHC class II complexes are shown, but similar events can occur for presentation of EV-derived peptides on MHC class I for presentation to CD8 ${ }^{+} \mathrm{T}$ cells.

cell surface for an extended period of time. The binding of EVs to FDCs is likely mediated in part through cell surface adhesion molecules, such as milk fat globule-EGF factor 8 (MFG-E8), ICAM1, and complement receptors 1 and 2 (CR1/2), which bind to EVexpressed phosphatidylserine (PS), CD11a, and C3-derived fragments, respectively (20).

A portion of EVs can be internalized by APCs after binding to the cell surface. Here, the EV-derived peptide/MHC complexes can be degraded and used as a source of peptide for presentation to $\mathrm{T}$ cells $(17,21)$. For example, HLA-DR $4^{+}$human EVs loaded with a serum albumin peptide were only able to stimulate $T$ cells following internalization by APCs that were also positive for HLA-DR4 (22). It is important to note that immature DCs internalize EVs more efficiently than mature DCs, suggesting that mature DCs are more likely to be involved in cross-dressing (17). In addition, because mature DCs are more stimulatory, cross-dressing is more likely to lead to immune stimulation than to immune suppression.

Peptides also can be directly loaded onto the vesicle-MHC complex (Figure 1B). For example, tumor antigen peptides, including peptides derived from the model tumor antigen ovalbumin (OVA), loaded onto EVs by pulsing can stimulate primed $\mathrm{T}$ cells as well as $\mathrm{T}$ cell clones, lines, and hybrids $(14,15,23,24)$. Similarly, viral peptides presented after peptide pulsing of the EVs can activate primed human peripheral $\mathrm{CD} 8^{+} \mathrm{T}$ cells, albeit at a low percentage. In contrast, EVs are not as effective in stimulating naive $\mathrm{T}$ cells. It appears as if the $\mathrm{T}$ cell stimulatory activity of primed $\mathrm{T}$ cells by EVs is significantly less efficient than that of the parent APCs. These results are consistent with a model in which the small size of EVs limits $\mathrm{T}$ cell receptor activation and costimulation. Indeed, mobilization of a high concentration of EVs on latex beads results in a markedly higher level of $\mathrm{T}$ cell stimulation, likely due to high concentrations of MHC complexes and costimulatory molecules $(22,25)$.

In addition, EVs are able to deliver native antigens to APCs by internalization and processing. The first example of this was in the context of delivery of tumor antigens by tumor-derived EVs (26, 27). Here, the tumor antigens in EVs are taken up by APCs and processed and cross-presented to cytotoxic $\mathrm{CD} 8^{+} \mathrm{T}$ cells. Moreover, vaccination of mice with tumor-derived exosomes was shown to induce a potent $\mathrm{CD} 8^{+} \mathrm{T}$ cell-mediated antitumor effect against not only the parental tumor, but also other tumors expressing similar tumor antigens (26). An important aspect of this cross-priming is that it is independent of the class of MHC molecules present on the EV. Additionally, EVs likely carry multiple tumor antigens, including unknown tumor antigens. In some cases, the tumor EVs appear to be enriched for a particular tumor antigen compared with the parental tumor cells. For example, certain tumor-derived EVs carry high levels of tumor antigens, such as melan-A, carcinoembryonic antigen (CEA), and/or mesothelin, compared with the parental tumor cells. Therefore, tumor-derived EVs can be used as a source of multiple tumor antigens to stimulate antitumor responses, both therapeutically or prophylactically. Treatment of DCs with tumor-derived EVs resulted in DCs that are able to induce CD8 $8^{+}$ $\mathrm{T}$ cell-dependent antitumor effects in mice and in patients with malignant gliomas. However, as discussed below, tumor-derived EVs also can carry additional immunoregulatory molecules on their surface, such as FasL, TRAIL, CD154 (also known as CD4OL), and programmed death-ligand 1 (PD-L1), which can reduce the immune response to tumor antigens, resulting in suppression of antitumor antigen-specific responses (28-32). 
A

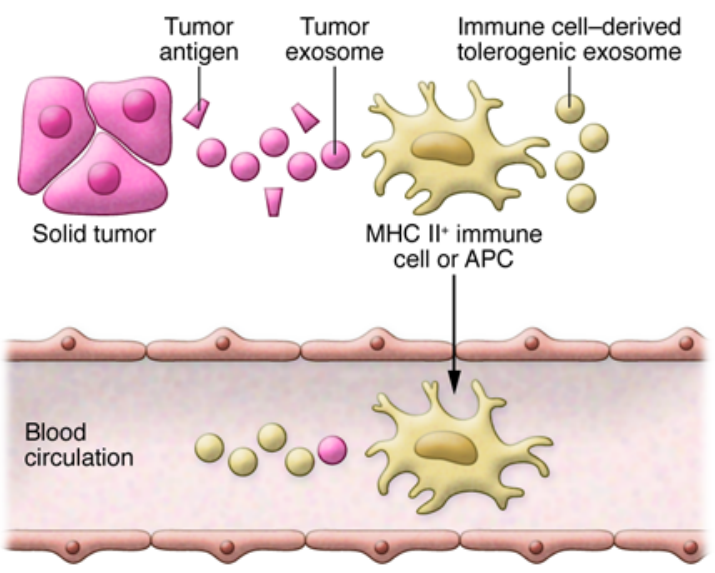

B

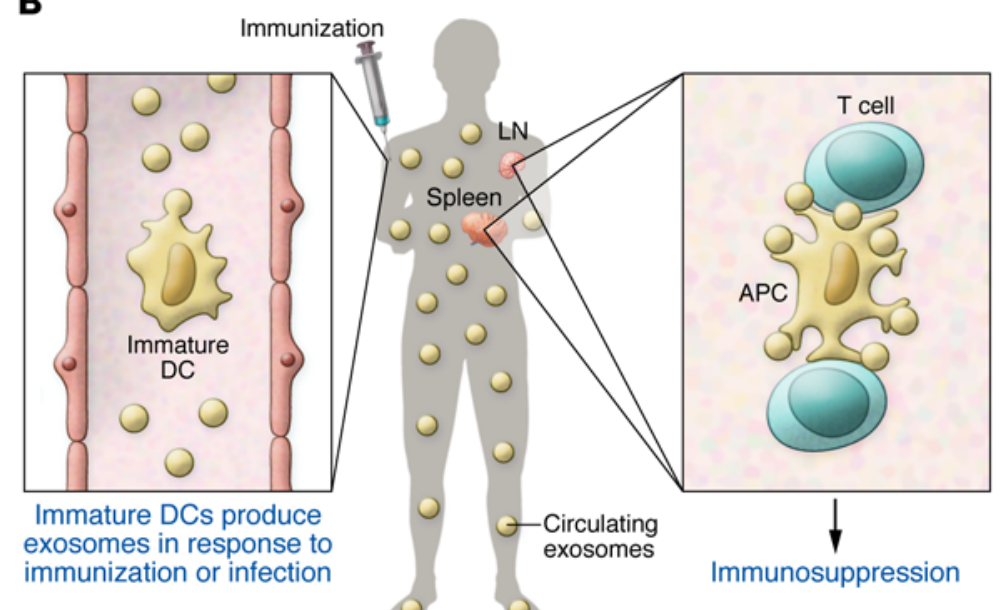

Figure 2. Role of endogenous EVs in regulating immune responses. (A) Resident APCs, such as macrophages in the tumor environment, can acquire tumor antigens either by uptake of tumor EVs or by phagocytosis of necrotic tumor cells. APCs then release MHC class II+ EVs into the circulation that are able to interact with immune cells at distant sites to suppress tumor antigen-specific immune responses; these are termed tolerogenic EVs. (B) Infection with a pathogen or immunization to an antigen results in acquisition of antigen by local APCs, followed by release of APC-derived EVs into the circulation. APC-derived EVs can carry the antigen and/or present antigen-derived epitopes in MHC class I and MHC class II complexes. Circulating EVs are taken up by APCs in the spleen or lymph nodes where they can modulate immune response to the antigen. It is hypothesized that these vesicles act to suppress the systemic immune response to the antigen or infection to limit the extent of inflammation or autoimmunity.

In addition to tumor antigens, antigens from both bacterial and viral pathogens can be delivered to APCs via EVs. For example, EVs derived from Mycobacterium bovis Bacillus Calmette Guérininfected macrophages promote $\mathrm{T}$ cell immunity in mice in the presence of DCs, whereas EVs from Toxoplasma gondii-pulsed DCs can confer protection against parasite infection in mice (33-35). EVs from CMV-infected epithelial cells stimulate memory CD $4^{+} \mathrm{T}$ cells in a DC-dependent manner. Viral antigens, such as EBV LMP-1 and influenza HA proteins, also are found in EVs and can contribute to regulating immune responses to these viruses $(35,36)$.

\section{Suppression of immune responses by EVs}

Although there is extensive evidence of the stimulation of immune responses by antigen-containing EVs, the release of EVs by tumor cells is one mechanism through which tumors suppress the antitumor immune response. Injection of tumor-derived EVs carrying the model tumor antigen OVA results in suppression of a delayed-type hypersensitivity (DTH) response to OVA (37). It is likely that the tumor EVs are taken up by endogenous APCs, which suppress the immune response following footpad injection of OVA. Similarly, injection of tumor-derived EVs inhibited the differentiation of $\mathrm{CD}_{11} \mathrm{~b}^{+}$myeloid precursors in the bone marrow $(\mathrm{BM})$, partially by inducing IL-6 expression in these precursor cells $(38,39)$. These myeloid cells also were shown to switch their differentiation pathway toward a myeloid-derived suppressor cell (MDSC) phenotype (40). Here, prostaglandin E2 (PGE2), HSP70, and certain miRNAs contained in the tumor-derived EVs play an important role in driving monocyte differentiation toward MDSCs. In addition to the effect on differentiation, there is also a concomitant increase in TGF- $\beta 1$ expression by myeloid cells, contributing to non-antigen-specific immunosuppression (41).

Tumor-derived EVs also can directly suppress the activity of effector T cells. In particular, certain tumor cell lines produce EVs expressing death ligands, such as FasL and TRAIL, which are both known to trigger the apoptotic death of activated $\mathrm{T}$ cells. They also can carry PD-L1 and CD4OL, which suppress $\mathrm{T}$ cell responses. Moreover, ovarian tumor-derived EVs modulate CD3- $\zeta$ chain expression, impairing $\mathrm{T}$ cell receptor signaling (42). In addition, NK cell and $\mathrm{CD} 8^{+} \mathrm{T}$ cell cytotoxicity was impaired by EVs containing ligand for the C-type lectin-like receptor NKG2D derived from certain human tumor types, such as breast cancer. Similarly, murine mammary carcinoma-derived EVs can promote tumor growth in vivo by suppressing NK cell function. Taken together, these observations suggest that tumor-derived EVs negatively regulate the effector arm of the immune system, in particular T cells and NK cells. However, it is likely in most, if not all, of these examples that immunosuppression mediated by tumor-derived EVs requires a very high dose of EVs or the involvement of APCs for cross-dressing.

Interestingly, EVs isolated from the sera of tumor-bearing mice can suppress the immune response to a specific tumor antigen through a MHC class II-dependent mechanism (43). Here, the suppression of antigen-specific immune response is mediated not by tumor-derived EVs, but by $\mathrm{CD}_{11} \mathrm{~b}^{+}, \mathrm{MHC}$ class $\mathrm{II}^{+}$host-derived EVs. Thus, it has been hypothesized that, within the tumor microenvironment, tumor EVs are taken up by macrophages and possibly other APCs, which in turn release EVs able to suppress immune responses to the tumor antigens present in the tumor EVs (Figure $2 \mathrm{~A}$ and ref. 43). Taken together, these results suggest that, not only can tumor-derived EVs directly suppress T and NK immune responses, but they also can suppress responses indirectly through macrophage cross-presentation and subsequent release of EVs.

In addition to suppression of tumor-specific immune responses mediated by tumor-derived and APC-derived EVs from the tumor microenvironment, tumor-derived vesicles also play a role in facilitating tumor invasion and metastasis (37) by establishing the premetastatic niche through the generation of a suitable 
microenvironment at potential metastatic sites. For example, melanoma-derived EVs preferentially home to sentinel lymph nodes where they facilitate the formation of tumor niches for metastatic tumor growth (44). Thus, tumor-derived EVs can function at multiple levels to facilitate tumor growth and metastasis.

As mentioned above, EVs have been found to contain functional mRNAs and small noncoding RNAs, including miRNAs, but generally not DNA or ribosomal RNAs $(6,45)$. Interestingly, the RNAs sorted into EVs, in particular exosomes, represent a subset of the parental RNA (46). In some cases, specific factors, such as sumoylated heterogeneous nuclear ribonucleoprotein A2/ B1 (hnRNPA2B1), mediate the targeting of particular miRNAs into vesicles $(47,48)$, suggesting that EV-associated RNA has specific functions in mediating cell-to-cell communication, especially in immune regulation. Indeed, EVs from DCs can transfer functional miRNAs to target cells, whereas EVs (49) from T cells transfer miRNA unidirectionally to DCs (45).

\section{Immune regulation by circulating, endogenous EVs}

In many situations, the main function of circulating, endogenous EVs is to suppress the immune response, either in an antigen-specific or a nonspecific manner. In one of the first examples of immunosuppression by endogenous exosomes, it was demonstrated that MHC class $\mathrm{II}^{+}$EVs isolated from the serum could suppress OVA-specific immune responses following oral administration of OVA (43). These serum EVs, initially termed tolerosomes, had markers indicating that they were derived from intestinal epithelial cells, which had acquired the digested OVA (43). Similar results were also obtained following intradermal or intranasal inoculation of antigen. For example, bronchoalveolar lavage fluid from mice repetitively immunized to the allergen Ole E1 by intranasal inoculation prevented allergic reactions to the same antigen (10, 50). Furthermore, following intradermal immunization with OVA, MHC class $\mathrm{II}^{+}$EVs could be isolated from serum that suppress an OVA-induced, but not an unrelated keyhole limpet hemocyanin-induced, DTH response in the footpad (51). These OVA-specific EVs also require FasL for their suppressive effects, although no increase in $\mathrm{T}$ cell apoptosis was detected (51). Surprisingly, the number and/or activity of OVA-specific, immunosuppressive EVs continues to increase in blood following antigen immunization, peaking at 14 days after immunization, with OVA-specific immunosuppressive activity detected in the serum at even 1 month (51) after inoculation. However, it is unclear whether APCs at the site of immunization or in the lymph node continue to release suppressive EVs for a long period of time and/or whether the circulating vesicles are highly stable. Given the observed antigen-specific suppressive activity of circulating EVs following intradermal immunization or oral delivery and in tumor-bearing mice, it has been proposed that the main function of blood-borne, APC-derived EVs is to suppress an active immune response to antigens (Figure 2B). This suppression by EVs would have the benefit of preventing self and foreign antigens from causing systemic, chronic inflammation and/or autoimmunity.

Other sources of endogenous EVs also negatively regulate immune responses. EVs with the ability to increase the number of Tregs can be isolated from breast milk $(52,53)$ and colostrum.
It is also well known that pregnancy reduces the severity of autoimmune diseases, such as rheumatoid arthritis (RA) and multiple sclerosis (54) (MS), in humans. Furthermore, EVs isolated from the serum of pregnant mice reduced the severity of experimental autoimmune encephalitis (EAE), a mouse model of MS (54). This immunosuppression is theorized to be mediated by placentalderived EVs, likely from trophoblasts, which are able to inhibit $\mathrm{T}$ cell signaling (55).

The immune response also is regulated by EVs from vascular ECs, while, in turn, immune cell EVs regulate the vascular endothelium. In particular, the inflammatory status of the vascular can affect the composition of the secreted EVs, thus allowing ECderived EVs (EC-EVs) to differentially modulate target cell responses in a paracrine fashion. For example, EC-EVs from quiescent cells reduced the proinflammatory response of cultured macrophages challenged with LPS, an antiinflammatory effect that was also observed in vivo. The antiinflammatory effect of EC-EVs was modulated, at least in part, through the transfer of miRNA10a, which inhibits NF- $\kappa \mathrm{B}$ signaling. EC-EVs from quiescent cells also may function in an autocrine fashion to maintain EC quiescence, which is protective against pathologies such as cardiovascular disease (56). In contrast, vascular ECs in an inflammatory environment release EVs that act in a paracrine manner to "educate" pericytes to promote angiogenesis via miRNA-mediated inhibition of YY1 and subsequent increase in VEGF production. Here, the inflammation-induced angiogenic phenotype mediated by the EC-EVs could enhance local inflammation, resulting in pathological vascular changes (57). Interestingly, treatment with endothelial progenitor cell-derived EVs (EPC-EVs) causes HUVECs and HMECs to organize into vessel-like structures both in vitro and in SCID mice via activation of the PI3/AKT and eNOS pathways (58). The EPC-EVs activate an angiogenic program in quiescent ECs, though it still remains to be determined how the inflammatory environment affects EPC-EV secretion, composition, and function.

In addition to inflammation of parent tissue, certain states of damage and exogenous factors that exacerbate pathologies in parent tissue can affect EV signaling. For example, hepatocytes increase their shedding of EVs in response to treatment with ethanol, an effect observed in vivo in a mouse model of chronic ethanol consumption. Furthermore, treatment of hepatocytes with ethanol results in release of EVs enriched with miRNA122. Following EV-mediated transfer of miRNA122 to monocytes, the monocytes become highly sensitive to LPS, upregulating their secretion of IL- $1 \beta$ and TNF- $\alpha$ compared with that after LPS treatment alone. This EV-mediated sensitization of monocytes could promote a state of chronic, systemic inflammation that further exacerbates the diagnosis phenotype and comorbidities in patients with liver disease $(59,60)$.

As mentioned above, gut epithelial cells release $\mathrm{MHC}$ class $\mathrm{II}^{+}$ EVs that are able to suppress an immune response to OVA following oral delivery of OVA. Additionally, EVs from different sources of MSCs appear to have not only immunoregulatory, but also regenerative, effects. Many types of MSCs, including BM- and fat tissue-derived MSCs, confer therapeutic effects in animal models of disease and tissue injury. In addition, human clinical trials have demonstrated that MSCs confer immunosuppressive effects for immune diseases such as Crohn's disease and graft-versus-host disease. Indeed, there are currently over 500 clinical trials using 


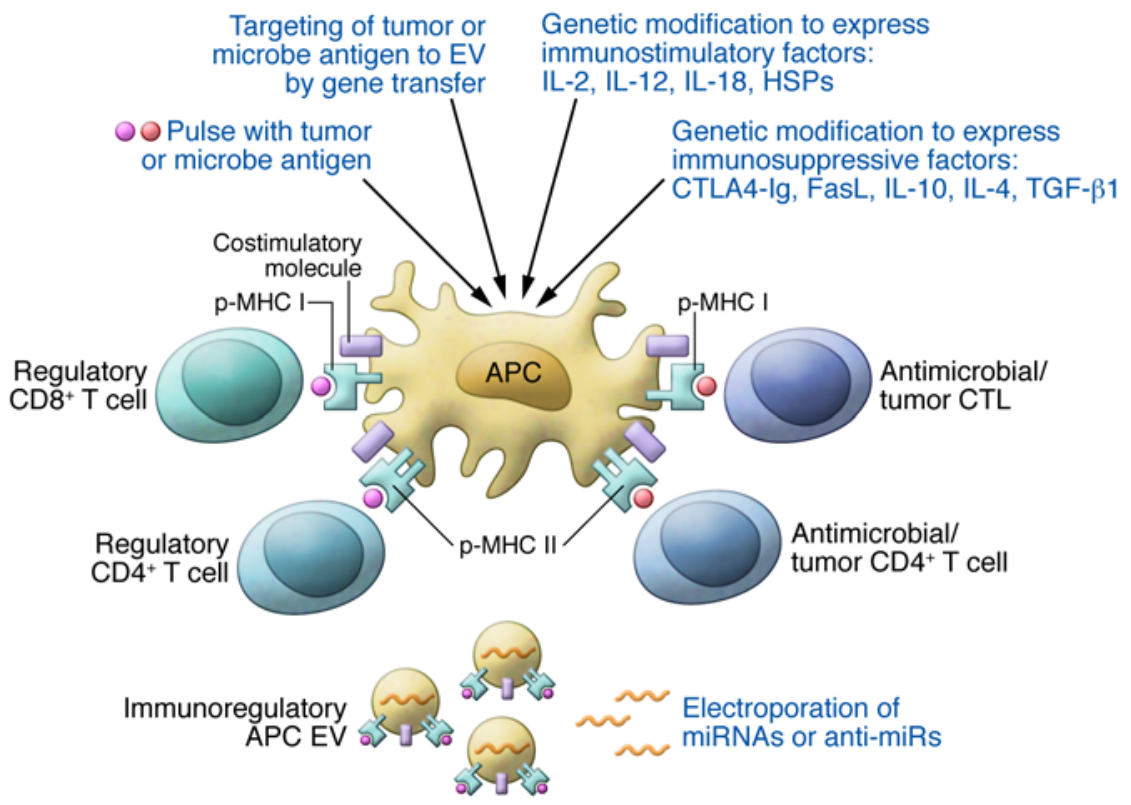

\begin{abstract}
Figure 3. Modifying EVs for therapeutic applications. APCs can be engineered in culture to generate immunoregulatory EVs for therapeutic applications. Antigen-specific effects can be achieved by pulsing the APCs with tumor- or pathogen-derived antigens or by transfer of antigen-encoding genes. Similarly, APCs can be modified to express immunosuppressive or immunostimulatory cytokines or ligands, which can render the APC-derived EVs able to suppress or stimulate antigen-specific immune responses. The expression of costimulatory ligands in the APC can result in EVs with increased levels of the ligands, thereby directly affecting the immunoregulatory activity of the EVs. Finally, the EVs themselves can be modified to carry immunomodulatory small RNAs, such as miRNAs or antagomIRs. CTL, cytotoxic T lymphocyte.
\end{abstract}

MSCs therapeutically for immunosuppression or tissue regeneration. It appears as if, in all or at least most of the preclinical studies with MSCs, the MSC therapeutic effects are conferred through a paracrine/endocrine mechanism mediated by immunosuppressive factors or growth factors $(61,62)$. Interestingly, MSC-derived EVs appear able to facilitate tissue regeneration as well as reduce inflammation $(55,63-66)$. Thus, many of the observed therapeutic effects of MSCs may be mediated, at least in part, by EVs.

\section{The role of EVs in autoimmunity}

In contrast to the ability of circulating EVs to suppress immune responses, endogenous EVs also appear to have a role in mediating inflammatory and autoimmune diseases. For example, EVs are a source of self antigens, including MHC-displayed self-peptides, that could activate autoreactive T cells $(9,67)$. In addition, the participation of EVs in immune complexes could stimulate autoimmunity. For example, EVs present in synovial fluid of patients with RA not only contain citrullinated autoantigens, but also can be found in complexes with anti-citrullinated peptide antibodies (68). Similarly, plasma EVs from systemic lupus erythematous (SLE) were shown to not only contain self antigens $(69,70)$, but also to interact with $\operatorname{IgG}$, IgM, and complement proteins. However, it was not clear whether these interactions represent true immune complexes (71-73). Additionally, EVs enhance autoimmune phenotypes through secretion or surface expression of proinflammatory cytokines that promote an inflammatory environment and autoreactive T cell survival $(74,75)$. For example, EVs in the sera and derived from synovial fibroblasts of patients with RA have higher levels of a membrane-bound form of TNF, a key therapeutic target in the treatment of RA, than EVs from healthy controls. Here, the $\mathrm{TNF}^{+}$EVs appear to confer resistance to apoptosis to activated $\mathrm{T}$ cells, in contrast to the ability of TNF to induce apoptosis, contributing to the T cell-mediated pathogenesis of RA (76).

In addition, circulating EVs may play a role in MS pathology, since there is an upregulation of endothelial, leukocyte, and platelet EVs with MS progression and a concomitant increase in metal- loproteinase activity in the EVs (77-79). These MS EVs could be at least partially responsible for the initial breakdown of the bloodbrain barrier that is necessary for MS. The type and amount of EVs present in patient sera also could promote survival of immune cells following CNS infiltration (80). In a similar manner, an increase in the amount of proinflammatory, procoagulant platelet-derived EVs in patients with Crohn's disease compared with that in healthy subjects was observed, implicating these EVs in disease progression (80). Both neutrophil-derived and platelet-derived EVs were shown to increase in patients with an anti-neutrophil cytoplasmic antibody-associated vasculitis (AAV) compared with that in remittent AAV subjects (81-83). Furthermore, platelet-derived EVs associated with oxidized high-mobility group box 1 protein (HMGB1) were shown to increase neutrophil activation, which could be responsible for phenotypes associated with systemic sclerosis. Here, EVs from healthy donors that did not contain HMGB1 failed to activate neutrophils, whereas treatment with HMGB1 inhibitors reversed the phenotypes that were potentially conferred by the systemic sclerosis EVs $(84,85)$.

\section{Therapeutic applications of EVs}

The ability of different types of EVs to modulate immune responses suggests they could be used therapeutically for a broad spectrum of diseases. In addition, any therapeutic application of EVs requires isolation of EVs from a specific cell source, allowing for modification of the parental cell to increase the immunoregulatory effects of the EVs. The best examples thus far are DC-derived exosomes in which the DC can be modified to alter the resulting EV. The DCs can be grown under certain conditions that maintain them in an immature or mature state, resulting in EVs that are more immunosuppressive or, through increased expression of costimulatory and MHC complexes, more stimulatory (86). For example, growing BM-derived APCs in high concentrations of IL-10, IL-4, or TGF- $\beta 1$, all of which promote an immature state, resulted in both DCs and EVs that suppressed inflammation in a DTH footpad model $(87,88)$ through MCH class II-, FasL-, and B7-dependent, but MHC class I- 
independent, mechanisms (88-90). Interestingly, treatment with DC-derived EVs in one footpad suppressed the DTH response in the contralateral footpad in an antigen-dependent manner. In this case, exogenous DC-derived EVs are able to modify the function of endogenous APCs in the footpad, which then traffic to the lymph node to modulate $\mathrm{T}$ cell responses. Immune regulatory factors, including but not limited to cytokines, can be directly delivered to the DC, where they alter the function of the DC and, thus, DCderived EVs. For example, gene transfer of a membrane-bound form of IL-4, FasL, or indoleamine-pyrrole 2,3-dioxygenase (IDO) to DCs results in both DCs and DC-derived EVs that are able to suppress early-stage disease in a murine model of collageninduced arthritis $(88,89,91)$. EVs expressing elevated levels of FasL on their surface confer their immune suppressive effects through a poorly understood Fas-dependent mechanism in the treated mice, although no increase in T cell apoptosis was observed (90). Furthermore, EVs derived from a stimulated B lymphocyte line that were directly loaded with an antagomIR-targeting miRNA155 were shown to reduce the inflammatory signaling of cultured macrophages being challenged with LPS. Thus, generating EVs containing miRNA or anti-miRs is a viable tool for enhancing the therapeutic activity of EVs (Figure 3).

In addition to enhancing the immunosuppressive effects of EVs, approaches to improve the immunostimulatory activity of EVs also are being developed. Several clinical trials using tumor EVs to load DCs with tumor antigen have been conducted with limited success $(27,92,93)$. However, the induction of a $\mathrm{CD}^{+}$cytotoxic $\mathrm{T}$ lymphocyte response against tumor cells was noted in a clinical study of malignant glioma, demonstrating the validity of the approach if the EV-treated DCs can be made immunostimulatory (94). For example, treatment of DCs with proinflammatory cytokines, such as IFN- $\gamma$ or IL-12, can result in enhanced stimulatory capacity (39, 95). Alternatively, gene transfer of cytokines or heat shock proteins to tumor cells can result in tumor-derived EVs that elicit a stronger DC-mediated immune response than unaltered tumor cells (85). Similarly, heat shock of tumor cells also enhances the stimulatory capacity of EVs, likely due to increased levels of heat shock proteins within the EVs $(96,97)$. Interestingly, the presence of antigens on the surface of the EV allows for induction of a stronger immune response than if the antigen is contained within the EV (98).

In addition to the use of EVs as therapeutics, proteins and RNAs carried in EVs could be used as biomarkers of immune function. The contents of EV subsets present in patient biofluids, including serum, urine, semen, saliva, and bronchoalveolar lavage fluid, easily could be used as noninvasive biomarkers or indicators of the host's immune status (99). For example, it was determined via flow cytometry that sera from patients with chronic hepatitis $\mathrm{C}$ have elevated levels of EVs from $\mathrm{CD}^{+}$and $\mathrm{CD} 8^{+} \mathrm{T}$ cells, whereas patients with nonalcoholic fatty liver or nonalcoholic steatohepatitis have elevated levels of EVs from iNKT cells and CD14 ${ }^{+}$macrophages/monocytes. The levels of these EVs in the sera correlated with disease severity and are thus a viable diagnostic tool for the assessment of these liver inflammatory diseases $(100,101)$. Furthermore, the RNA profile of urine EVs from patients with SLE suggests that this approach could be used to determine the extent of kidney damage from lupus nephritis, which causes SLE-related morbidity and mortality $(102,103)$.

\section{Summary}

The EVs released by many cell types play important roles in cellto-cell communication, especially in regard to immune regulation. EVs derived from both immune and nonimmune cells carry self antigens following infection by pathogens and other foreign antigens. In this context, endogenous EVs likely modulate immune responses, serving to stimulate immune responses to foreign antigens, while suppressing the response to self antigens. Although EVs can modulate the function of many immune cell types, including $\mathrm{T}$ and NK cells (albeit weakly), the most effective regulatory activity of EVs is conferred through APCs, either by binding to the cell surface or by internalization. A better understanding of how EVs from different immune cell types function in vivo to regulate immunity should provide new insights into how the immune system communicates as well as aid in the development of better therapeutics. In the context of cancer, the reduction in release of EVs by the tumor or depletion of tumor exosomes from the circulation should result in a stronger antitumor response while reducing the risk of metastasis. For treatment of autoimmune diseases, modified APC-derived EVs or even endogenous EVs isolated from the circulation of patients could be used therapeutically. A key advantage to using EVs therapeutically is that, unlike the parental cells, EVs cannot change their phenotype: a suppressive $\mathrm{EV}$ remains a suppressive EV. Based on the strong immune regulation observed with both exogenous and endogenous EVs, it is likely that the next decade will see a large number of clinical studies based on EVs, either as the therapy or as the therapeutic target.

\section{Acknowledgments}

This work was supported by the public health grants AG024827, AG03307, AR051456, and AG043376 from the National Institutes of Health; a program grant from the Juvenile Diabetes Research Foundation; and Aldabra Biosciences.

Address correspondence to: Paul D. Robbins, Department of Metabolism and Aging, The Scripps Research Institute-Florida, 130 Scripps Way, Jupiter, Florida 33458, USA. Phone: 561.228.2126; E-mail:probbins@scripps.edu.
1. Thery C, Zitvogel L, Amigorena S. Exosomes: composition, biogenesis and function. Nat Rev Immunol. 2002;2(8):569-579.

2. EL Andaloussi S, Mäger I, Breakefield XO, Wood MJ. Extracellular vesicles: biology emerging therapeutic opportunities. Nat Rev Drug Discov. 2013;12(5):347-357.

3. Raposo G, Stoorvogel W. Extracellular vesicles: exosomes, microvesicles, and friends. J Cell Biol. 2013;200(4):373-383

4. Robbins PD, Morelli AE. Regulation of immune responses by extracellular vesicles. Nat Rev Immunol. 2014;14(3):195-208.

5. Ratajczak J, Wysoczynski M, Hayek F, Janowska-Wieczorek A, Ratajczak MZ. Membranederived microvesicles: important and underap- preciated mediators of cell-to-cell communication. Leukemia. 2006;20(9):1487-1495.

6. Valadi H, Ekstrom K, Bossios A, Sjostrand M, Lee JJ, Lotvall JO. Exosome-mediated transfer of mRNAs and microRNAs is a novel mechanism of genetic exchange between cells. Nat Cell Biol. 2007;9(6):654-659.

7. Balaj L, et al. Tumour microvesicles contain 
retrotransposon elements and amplified oncogene sequences. Nat Commun. 2011;2:180.

8. Dorronsoro A, et al. Human mesenchymal stromal cell-mediated immunoregulation: mechanisms of action and clinical applications. Bone Marrow Res. 2013;2013:203643.

9. Turpin D, et al. Role of extracellular vesicles in autoimmune diseases. Autoimmun Rev. 2016;15(2):174-183.

10. Prado N, et al. Exosomes from bronchoalveolar fluid of tolerized mice prevent allergic reaction. Jimmunol. 2008;181(2):1519-1525.

11. Thery C, et al. Molecular characterization of dendritic cell-derived exosomes. J Cell Biol. 1999;147(3):599-610.

12. Raposo G, et al. B lymphocytes secrete antigen-presenting vesicles. J Exp Med. 1996;183(3):1161-1172.

13. Buschow SI, et al. MHC II in dendritic cells is targeted to lysosomes or T cell-induced exosomes via distinct multivesicular body pathways. Traffic. 2009;10(10):1528-1542.

14. Nolte-'t Hoen EN, Buschow SI, Anderton SM, Stoorvogel W, Wauben MH. Activated T cells recruit exosomes secreted by dendritic cells via LFA-1. Blood. 2009;113(9):1977-1981.

15. Muntasell A, Berger AC, Roche PA. T cellinduced secretion of MHC class II-peptide complexes on B cell exosomes. EMBO J. 2007;26(19):4263-4272.

16. Wakim LM, Bevan MJ. Cross-dressed dendritic cells drive memory $\mathrm{CD} 8^{+} \mathrm{T}$-cell activation after viral infection. Nature. 2011;471(7340):629-632.

17. Montecalvo A, et al. Exosomes as a short-range mechanism to spread alloantigen between dendritic cells during $\mathrm{T}$ cell allorecognition. J Immunol. 2008;180(5):3081-3090.

18. Segura E, et al. ICAM-1 on exosomes from mature dendritic cells is critical for efficient naive T-cell priming. Blood. 2005;106(1):216-223.

19. Utsugi-Kobukai S, Fujimaki H, Hotta C, Nakazawa M, Minami M. MHC class I-mediated exogenous antigen presentation by exosomes secreted from immature and mature bone marrow derived dendritic cells. Immunol Lett. 2003;89(2-3):125-131.

20. El Shikh ME, Pitzalis C. Follicular dendritic cells in health and disease. Front Immunol. 2012;3:292.

21. Mallegol J, et al. T84-intestinal epithelial exosomes bear MHC class II/peptide complexes potentiating antigen presentation by dendritic cells. Gastroenterology. 2007;132(5):1866-1876.

22. Qazi KR, Gehrmann U, Domange Jordo E, Karlsson MC, Gabrielsson S. Antigen-loaded exosomes alone induce Th1-type memory through a B-cell-dependent mechanism. Blood. 2009;113(12):2673-2683.

23. Admyre C, et al. B cell-derived exosomes can present allergen peptides and activate allergen-specific $\mathrm{T}$ cells to proliferate and produce TH2-like cytokines. J Allergy Clin Immunol. 2007;120(6):1418-1424.

24. Admyre C, Johansson SM, Paulie S, Gabrielsson $\mathrm{S}$. Direct exosome stimulation of peripheral human T cells detected by ELISPOT. Eur J Immunol. 2006;36(7):1772-1781.

25. Hsu DH, et al. Exosomes as a tumor vac- cine: enhancing potency through direct loading of antigenic peptides. J Immunother. 2003;26(5):440-450.

26. Wolfers J, et al. Tumor-derived exosomes are a source of shared tumor rejection antigens for CTL cross-priming. Nat Med. 2001;7(3):297-303.

27. Andre F, et al. Malignant effusions and immunogenic tumour-derived exosomes. Lancet. 2002;360(9329):295-305.

28. Monleon I, et al. Differential secretion of Fas ligand- or APO2 ligand/TNF-related apoptosisinducing ligand-carrying microvesicles during activation-induced death of human T cells. Jimmunol. 2001;167(12):6736-6744.

29. Zuccato E, et al. Sorting of Fas ligand to secretory lysosomes is regulated by mono-ubiquitylation and phosphorylation. J Cell Sci. 2007; 120(pt 1):191-199.

30. Munich S, Sobo-Vujanovic A, Buchser WJ, BeerStolz D, Vujanovic NL. Dendritic cell exosomes directly kill tumor cells and activate natural killer cells via TNF superfamily ligands. Oncoimmunology. 2012;1(7):1074-1083.

31. Andreola G, et al. Induction of lymphocyte apoptosis by tumor cell secretion of FasL-bearing microvesicles. J Exp Med. 2002;195(10):1303-1316.

32. Frangsmyr L, Baranov V, Nagaeva O, Stendahl U, Kjellberg L, Mincheva-Nilsson L. Cytoplasmic microvesicular form of Fas ligand in human early placenta: switching the tissue immune privilege hypothesis from cellular to vesicular level. $\mathrm{Mol}$ Hum Reprod. 2005;11(1):35-41.

33. Giri PK, Schorey JS. Exosomes derived from $M$. Bovis BCG infected macrophages activate antigen-specific $\mathrm{CD}^{+}$and $\mathrm{CD} 8^{+} \mathrm{T}$ cells in vitro and in vivo. PLoS One. 2008;3(6):e2461.

34. Beauvillain C, Ruiz S, Guiton R, Bout D, Dimier-Poisson I. A vaccine based on exosomes secreted by a dendritic cell line confers protection against T. Microbes Infect. 2007;9(14-15):1614-1622.

35. Keryer-Bibens $\mathrm{C}$, et al. Exosomes released by EBV-infected nasopharyngeal carcinoma cells convey the viral latent membrane protein 1 and the immunomodulatory protein galectin 9. BMC Cancer. 2006;6:283.

36. Testa JS, Apcher GS, Comber JD, Eisenlohr LC. Exosome-driven antigen transfer for MHC class II presentation facilitated by the receptor binding activity of influenza hemagglutinin. JImmunol. 2010;185(11):6608-6616.

37. Costa-Silva B, et al. Pancreatic cancer exosomes initiate pre-metastatic niche formation in the liver. Nat Cell Biol. 2015;17(6):816-826.

38. Yu S, et al. Tumor exosomes inhibit differentiation of bone marrow dendritic cells. JImmunol. 2007;178(11):6867-6875.

39. Zhang Y, Luo CL, He BC, Zhang JM, Cheng G, $\mathrm{Wu}$ XH. Exosomes derived from IL-12anchored renal cancer cells increase induction of specific antitumor response in vitro: a novel vaccine for renal cell carcinoma. Int J Oncol. 2010;36(1):133-140.

40. Xiang X, et al. TLR2-mediated expansion of MDSCs is dependent on the source of tumor exosomes. Am J Pathol. 2010;177(4):1606-1610.

41. Valenti R, et al. Human tumor-released microves- icles promote the differentiation of myeloid cells with transforming growth factor- $\beta$-mediated suppressive activity on T lymphocytes. Cancer Res. 2006;66(18):9290-9298.

42. Taylor DD, Gercel-Taylor C, Lyons KS, Stanson J, Whiteside TL. T-cell apoptosis and suppression of T-cell receptor/CD3- $\zeta$ by Fas ligand-containing membrane vesicles shed from ovarian tumors. Clin Cancer Res. 2003;9(14):5113-5119.

43. Ostman S, Taube M, Telemo E. Tolerosomeinduced oral tolerance is MHC dependent. Immunology. 2005;116(4):464-476.

44. Peinado $\mathrm{H}$, et al. Melanoma exosomes educate bone marrow progenitor cells toward a prometastatic phenotype through MET. Nat Med. 2012;18(6):883-891.

45. Mittelbrunn M, et al. Unidirectional transfer of microRNA-loaded exosomes from T cells to antigen-presenting cells. Nat Commun. 2011;2:282.

46. Nolte-'t Hoen EN, Buermans HP, Waasdorp M, Stoorvogel W, Wauben MH, t Hoen PA. Deep sequencing of RNA from immune cell-derived vesicles uncovers the selective incorporation of small non-coding RNA biotypes with potential regulatory functions. Nucleic Acids Res. 2012;40(18):9272-9285.

47. Villarroya-Beltri C, et al. Sumoylated hnRNPA2B1 controls the sorting of miRNAs into exosomes through binding to specific motifs. Nat Commun. 2013;4:2980.

48. Villarroya-Beltri C, Baixauli F, Gutierrez-Vazquez C, Sanchez-Madrid F, Mittelbrunn M. Sorting it out: regulation of exosome loading. Semin Cancer Biol. 2014;28:3-13.

49. Montecalvo A, et al. Mechanism of transfer of functional microRNAs between mouse dendritic cells via exosomes. Blood. 2012;119(3):756-766.

50. Admyre C, et al. Exosomes with major histocompatibility complex class II and co-stimulatory molecules are present in human BAL fluid. Eur Respir J. 2003;22(4):578-583.

51. Kim SH, Bianco NR, Shufesky WJ, Morelli AE, Robbins PD. MHC class $\mathrm{II}^{+}$exosomes in plasma suppress inflammation in an antigen-specific and Fas ligand/Fas-dependent manner. J Immunol. 2007;179(4):2235-2241.

52. Admyre C, et al. Exosomes with immune modulatory features are present in human breast milk. Jimmunol. 2007;179(3):1969-1978.

53. Zhou Q, et al. Immune-related microRNAs are abundant in breast milk exosomes. Int J Biol Sci. 2012;8(1):118-123.

54. Gatson NN, et al. Induction of pregnancy during established EAE halts progression of CNS autoimmune injury via pregnancy-specific serum factors. J Neuroimmunol. 2011;230(1-2):105-113.

55. Arslan F, et al. Mesenchymal stem cell-derived exosomes increase ATP levels, decrease oxidative stress and activate PI3K/Akt pathway to enhance myocardial viability prevent adverse remodeling after myocardial ischemia/reperfusion injury. Stem Cell Res. 2013;10(3):301-312.

56. Njock MS, et al. Endothelial cells suppress monocyte activation through secretion of extracellular vesicles containing antiinflammatory microRNAs. Blood. 2015;125(20):3202-3212.

57. Yamamoto S, et al. Inflammation-induced endothelial cell-derived extracellular vesicles 
modulate the cellular status of pericytes. Sci Rep. 2015;5:8505.

58. Deregibus MC, et al. Endothelial progenitor cell derived microvesicles activate an angiogenic program in endothelial cells by a horizontal transfer of mRNA. Blood. 2007;110(7):2440-2448.

59. Momen-Heravi F, Saha B, Kodys K, Catalano D, Satishchandran A, Szabo G. Increased number of circulating exosomes and their microRNA cargos are potential novel biomarkers in alcoholic hepatitis. J Transl Med. 2015;13:261.

60. Momen-Heravi F, Bala S, Kodys K, Szabo G. Exosomes derived from alcohol-treated hepatocytes horizontally transfer liver specific miRNA122 and sensitize monocytes to LPS. Sci Rep. 2015;5:9991.

61. Caplan AI, Correa D. The MSC: an injury drugstore. Cell Stem Cell. 2011;9(1):11-15.

62. Pittenger M. Sleuthing the source of regeneration by MSCs. Cell Stem Cell. 2009;5(1):8-10.

63. Bruno S, et al. Microvesicles derived from mesenchymal stem cells enhance survival in a lethal model of acute kidney injury. PLoS One. 2012;7(3):e33115.

64. Cantaluppi V, et al. Microvesicles derived from endothelial progenitor cells protect the kidney from ischemia-reperfusion injury by microRNAdependent reprogramming of resident renal cells. Kidney Int. 2012;82(4):412-427.

65. Lai RC, et al. Exosome secreted by MSC reduces myocardial ischemia/reperfusion injury. Stem Cell Res. 2010;4(3):214-222.

66. Lai RC, Yeo RW, Tan KH, Lim SK. Mesenchymal stem cell exosome ameliorates reperfusion injury through proteomic complementation. Regen Med. 2013;8(2):197-209.

67. Buzas EI, Gyorgy B, Nagy G, Falus A, Gay S. Emerging role of extracellular vesicles in inflammatory diseases. Nat Rev Rheumatol. 2014;10(6):356-364.

68. Cloutier N, et al. The exposure of autoantigens by microparticles underlies the formation of potent inflammatory components: the microparticleassociated immune complexes. ЕMBO Mol Med. 2013;5(2):235-249.

69. Dieker J, et al. Circulating apoptotic microparticles in systemic lupus erythematosus patients drive the activation of dendritic cell subsets and prime neutrophils for NETosis. Arthritis Rheumatol. 2016;68(2):462-472.

70. Dye JR, Ullal AJ, Pisetsky DS. The role of microparticles in the pathogenesis of rheumatoid arthritis and systemic lupus erythematosus. Scand J Immunol. 2013;78(2):140-148.

71. Nielsen CT, Ostergaard O, Johnsen C, Jacobsen S, Heegaard NH. Distinct features of circulating microparticles and their relationship to clinical manifestations in systemic lupus erythematosus. Arthritis Rheum. 2011;63(10):3067-3077.

72. Nielsen CT, et al. Increased IgG on cell-derived plasma microparticles in systemic lupus erythematosus is associated with autoantibodies and complement activation. Arthritis Rheum. 2012;64(4):1227-1236.

73. Ullal AJ, et al. Microparticles as antigenic tar- gets of antibodies to DNA and nucleosomes in systemic lupus erythematosus. J Autoimmun. 2011;36(3-4):173-180.

74. Pizzirani C, et al. Stimulation of $\mathrm{P} 2$ receptors causes release of IL-1beta-loaded microvesicles from human dendritic cells. Blood. 2007;109(9):3856-3864.

75. Boilard E, et al. Platelets amplify inflammation in arthritis via collagen-dependent microparticle production. Science. 2010;327(5965):580-583.

76. Zhang HG, et al. A membrane form of TNFalpha presented by exosomes delays $\mathrm{T}$ cell activation-induced cell death. Jimmunol. 2006;176(12):7385-7393.

77. Lo Cicero A, Majkowska I, Nagase H, Di Liegro I, Troeberg L. Microvesicles shed by oligodendroglioma cells and rheumatoid synovial fibroblasts contain aggrecanase activity. Matrix Biol. 2012;31(4):229-233.

78. Saenz-Cuesta M, et al. Circulating microparticles reflect treatment effects and clinical status in multiple sclerosis. Biomark Med. 2014;8(5):653-661.

79. Saenz-Cuesta M, Osorio-Querejeta I, Otaegui D. Extracellular vesicles in multiple sclerosis: what are they telling us? Front Cell Neurosci. 2014;8:100.

80. Ardoin SP, Shanahan JC, Pisetsky DS. The role of microparticles in inflammation and thrombosis. Scand J Immunol. 2007;66(2-3):159-165.

81. Kambas K, et al. Tissue factor expression in neutrophil extracellular traps and neutrophil derived microparticles in antineutrophil cytoplasmic antibody associated vasculitis may promote thromboinflammation and the thrombophilic state associated with the disease. Ann Rheum Dis. 2014;73(10):1854-1863.

82. Apostolidou E, et al. Neutrophil extracellular traps regulate IL-1beta-mediated inflammation in familial Mediterranean fever. Ann Rheum Dis. 2016;75(1):269-277.

83. Hong Y, et al. Anti-neutrophil cytoplasmic antibodies stimulate release of neutrophil microparticles. J Am Soc Nephrol. 2012;23(1):49-62.

84. Maugeri N, et al. Activated platelets present high mobility group box 1 to neutrophils, inducing autophagy promoting the extrusion of neutrophil extracellular traps. J Thromb Haemost. 2014;12(12):2074-2088

85. Maugeri N, et al. Oxidative stress elicits platelet/leukocyte inflammatory interactions via HMGB1: a candidate for microvessel injury in sytemic sclerosis. Antioxid Redox Signal. 2014;20(7):1060-1074.

86. Thomson AW, Robbins PD. Tolerogenic dendritic cells for autoimmune disease and transplantation. Ann Rheum Dis. 2008;67(suppl 3):iii90-iii96.

87. Kim SH, et al. Exosomes derived from IL-10treated dendritic cells can suppress inflammation collagen-induced arthritis. JImmunol. 2005;174(10):6440-6448.

88. Kim SH, Bianco NR, Shufesky WJ, Morelli AE, Robbins PD. Effective treatment of inflammatory disease models with exosomes derived from dendritic cells genetically modified to express IL-4.
JImmunol. 2007;179(4):2242-2249.

89. Ruffner MA, Kim SH, Bianco NR, Francisco LM, Sharpe AH, Robbins PD. B7-1/2, but not PD-L1/2 molecules, are required on IL-10-treated tolerogenic DC DC-derived exosomes for in vivo function. Eur J Immunol. 2009;39(11):3084-3090.

90. Kim SH, et al. Exosomes derived from genetically modified DC expressing FasL are anti-inflammatory and immunosuppressive. Mol Ther. 2006;13(2):289-300.

91. Bianco NR, Kim SH, Ruffner MA, Robbins PD. Therapeutic effect of exosomes from indoleamine 2,3-dioxygenase-positive dendritic cells in collagen-induced arthritis delayed-type hypersensitivity disease models. Arthritis Rheum. 2009;60(2):380-389.

92. Andre F, et al. Exosomes as potent cellfree peptide-based vaccine. J Immunol. 2004;172(4):2126-2136.

93. Escudier B, et al. Vaccination of metastatic melanoma patients with autologous dendritic cell (DC) derived-exosomes: results of the first phase I clinical trial. J Transl Med. 2005;3(1):10.

94. Bu N, et al. Exosome-loaded dendritic cells elicit tumor-specific $\mathrm{CD} 8{ }^{+}$cytotoxic $\mathrm{T}$ cells in patients with glioma. J Neurooncol. 2011;104(3):659-667.

95. Viaud S, et al. Updated technology to produce highly immunogenic dendritic cell-derived exosomes of clinical grade: a critical role of interferongamma. J Immunother. 2011;34(1):65-75.

96. Chen W, et al. Efficient induction of antitumor $\mathrm{T}$ cell immunity by exosomes derived from heat-shocked lymphoma cells. Eur JImmunol. 2006;36(6):1598-1607.

97. Dai S, et al. More efficient induction of HLA$A^{*}$ 0201-restricted and carcinoembryonic antigen (CEA)-specific CTL response by immunization with exosomes prepared from heat-stressed CEA-positive tumor cells. Clin Cancer Res. 2005;11(20):7554-7563.

98. Xiu F, Cai Z, Yang Y, Wang X, Wang J, Cao X. Surface anchorage of superantigen SEA promotes induction of specific antitumor immune response by tumor-derived exosomes. J Mol Med (Berl). 2007;85(5):511-521.

99. Revenfeld AL, Baek R, Nielsen MH, Stensballe A, Varming K, Jorgensen M. Diagnostic and prognostic potential of extracellular vesicles in peripheral blood. Clin Ther. 2014;36(6):830-846.

100. Julich H, Willms A, Lukacs-Kornek V, Kornek M. Extracellular vesicle profiling and their use as potential disease specific biomarker. Front Immunol. 2014;5:413.

101.Kornek M, et al. Circulating microparticles as disease-specific biomarkers of severity of inflammation in patients with hepatitis $\mathrm{C}$ or nonalcoholic steatohepatitis. Gastroenterology. 2012;143(2):448-458.

102. Perez-Hernandez J, Cortes R. Extracellular vesicles as biomarkers of systemic lupus erythematosus. Dis Markers. 2015;2015:613536.

103. Perez-Hernandez J, Forner MJ, Pinto C, Chaves FJ, Cortes R, Redon J. Increased urinary exosomal microRNAs in patients with systemic lupus erythematosus. PLoS One. 2015;10(9):e0138618. 